ARTICLE

\title{
Tunable solid-state fluorescent materials for supramolecular encryption
}

Xisen Hou ${ }^{1, \star}$, Chenfeng Ke $\mathrm{e}^{1, \star}$, Carson J. Bruns ${ }^{1}$, Paul R. McGonigal ${ }^{1}$, Roger B. Pettman ${ }^{2}$ \& J. Fraser Stoddart ${ }^{1}$

Tunable solid-state fluorescent materials are ideal for applications in security printing technologies. A document possesses a high level of security if its encrypted information can be authenticated without being decoded, while also being resistant to counterfeiting. Herein, we describe a heterorotaxane with tunable solid-state fluorescent emissions enabled through reversible manipulation of its aggregation by supramolecular encapsulation. The dynamic nature of this fluorescent material is based on a complex set of equilibria, whose fluorescence output depends non-linearly on the chemical inputs and the composition of the paper. By applying this system in fluorescent security inks, the information encoded in polychromic images can be protected in such a way that it is close to impossible to reverse engineer, as well as being easy to verify. This system constitutes a unique application of responsive complex equilibria in the form of a cryptographic algorithm that protects valuable information printed using tunable solid-state fluorescent materials.

\footnotetext{
${ }^{1}$ Department of Chemistry, Northwestern University, Evanston, Illinois 60208-3113, USA. ${ }^{2}$ Cycladex, c/o Innovation and New Ventures Office, Northwestern University, 1800 Sherman Avenue, Suite 504, Evanston, Illinois 60201-3789, USA. * These authors contributed equally to this work. Correspondence and requests for materials should be addressed to J.F.S. (email: stoddart@northwestern.edu).
} 
$\mathrm{P}$ hotoluminescent solid-state materials have been widely applied in dye lasers ${ }^{1}$, organic light emitting diodes (OLEDs) $^{2-4}$, data recording and storage ${ }^{5,6}$, and security printing ${ }^{7,8}$. These materials can be easily applied inexpensively to different surfaces and have been implemented widely as security inks to protect high-value merchandise, government documents and banknotes 9 . Materials with static luminescent outputs, however, are familiar to counterfeiters. In contrast, stimuliresponsive photoluminescent materials, which change their optical outputs in response to external stimuli, possess extra security features that are difficult to mimic, making them suitable for the next generation of security printing. In recent years, a series of stimuli-responsive photoluminescent materials, including those that are thermochromic ${ }^{10-13}$, photochromic ${ }^{14-16}$, mechanochromic $^{17-19}$, solvochromic ${ }^{20-23}$ and electrochromic ${ }^{24,25}$, have been developed. These smart materials respond to external stimuli with reversible changes to their chemical constitutions or superstructures in the solid state, causing them to emit different luminescent colours. Coding these fluorescent colours in one or two dimensions - a practice which is similar to digital coding (for example, barcodes and QR codes) in computer science- has been suggested $^{26}$ as a potential strategy to prevent tampering or counterfeiting. Current stimuli-responsive photoluminescent materials, however, can only provide a small matrix of colours as optical codes. Developing wide-spectrum tunable photoluminescent solid-state materials with multiple fluorescent emissions (a large matrix) remains a major challenge.

Herein, we report the unexpected discovery, during synthesis by a cooperative capture strategy ${ }^{27-29}$, of a hetero[4] rotaxane $\mathbf{R} 4 \bullet 4 \mathrm{Cl}$, which contains pyrene stoppers and a diazaperopyrenium unit, derived from $\mathbf{1} \bullet \mathrm{Cl}$ and $2 \bullet 2 \mathrm{Cl}$, respectively. Its fluorescent emission in the solid state can be fine-tuned rapidly and reversibly over a wide $(\sim 100 \mathrm{~nm})$ range of wavelengths as a result of stimuli-responsive aggregation and de-aggregation processes that are governed by a network of supramolecular equilibria. The unique features of this heterorotaxane include (i) the widely tunable colour of its fluorescent emission, providing a large analogue matrix of optical outputs, (ii) variations in fluorescent emission colour that occur when the heterorotaxane is deposited on different types of paper and (iii) the non-linear variation in its colour profile in response to simple chemical stimuli. These properties have allowed us to demonstrate a system of chemical encryption, in which the heterorotaxane is employed as a fluorescent security ink and its complex supramolecular equilibria serve as an encryption algorithm. By encrypting graphical information using these inks, printed images are nigh impossible to mimic, counterfeit and reverse engineer, yet can be easily verified on application of an appropriate authentication reagent, without revealing the original information.

\section{Results}

Synthesis of hetero[n]rotaxanes. The key compound in our investigations is the heterorotaxane $\mathbf{R} \mathbf{4} \bullet \mathbf{4 C l}$, which was isolated (Supplementary Figs $1-10$ ) as an unexpected side product during the synthesis (Fig. 1a) of the heterorotaxane $\mathbf{R} 3 \bullet 4 \mathrm{Cl}$ (Supplementary Figs $11-14$ ) from cucurbit[6]uril (CB6), $\gamma$-cyclodextrin $(\gamma$-CD) and two fluorescent precursors, one $(\mathbf{1} \bullet \mathrm{Cl})$ derived from pyrene and the other $(2 \bullet 2 \mathrm{Cl})$ from a diazaperopyrenium (DAPP) dication. In common with many fluorophores, DAPP exhibits a high fluorescence quantum yield ${ }^{30,31}$ in solution $(\Phi=53 \%)$ but not in the solid state $(\Phi=0 \%)$ as a result of aggregation-induced quenching. To 'turn on' the fluorescence of DAPP in the solid state, we attempted to de-aggregate ${ }^{32}$ DAPP by encapsulating $2 \bullet 2 \mathrm{Cl}$ with $\gamma$-CD. No complexation, however, between $2 \bullet 2 \mathrm{Cl}$ and $\gamma$-CD was observed (Fig. 1b) in aqueous solution (Supplementary Figs $15-17$ ). We anticipated that a more effective strategy would be to fix two bulky CB6 rings at the periphery of the DAPP dye as part of a mechanically interlocked molecule, formed by means of the highly efficient and rapid cooperative capture synthesis $^{27-29}$ in aqueous solution. To our surprise, the reaction not only afforded the anticipated heterorotaxane $\mathbf{R} 3 \bullet 4 \mathrm{Cl}$ (yield $=80 \%$ ), but also another heterorotaxane $\mathbf{R} 4 \bullet 4 \mathrm{Cl}$ (yield $=9 \%$ ). The formation of $\mathbf{R} 4 \bullet 4 \mathrm{Cl}$, in which $\gamma$-CD encircles the DAPP unit, seems to be at odds with the observation that $\gamma$-CD does not bind $2 \bullet 2 \mathrm{Cl}$. The unfavourable energetics of threading $\gamma$-CD onto the dumbbell of the heterorotaxane are outweighed by the positive contribution from the hydrogen bonding network formed between $\gamma$-CD and the neighbouring CB6, an observation which is supported by molecular mechanics simulations (Supplementary Fig. 43), as well as the release of high-energy water molecules ${ }^{33}$ from the macrocycles. Increasing the amount of $\gamma$-CD to 10 equiv. in the reaction mixture favours the formation of $\mathbf{R} 4 \bullet 4 \mathrm{Cl}$, which was isolated as the major product in $83 \%$ yield in $3 \mathrm{~h}$ (Supplementary Table 1). Although both heterorotaxanes dissolve to some extent in water, $\mathbf{R} 3 \bullet 4 \mathrm{Cl}$, which lacks a solubilizing $\gamma$-CD ring, is poorly soluble and undergoes aggregation. In contrast, $\mathbf{R} 4 \bullet 4 \mathrm{Cl}$ exhibits significant water solubility of up to $3 \mathrm{mM}$ at room temperature. ${ }^{1} \mathrm{H}$ NMR spectroscopy reveals that, while $\mathbf{R} 4 \bullet 4 \mathrm{Cl}$ undergoes aggregation (Fig. 1c and Supplementary Figs 18-19) at room temperature, it experiences de-aggregation to its monomeric form (Fig. 1d) on heating to $80^{\circ} \mathrm{C}$. 2D-NOESY and variable temperature NMR experiments indicate (Supplementary Figs $20-21)$ that the $\gamma-\mathrm{CD}$ ring in $\mathbf{R} 4 \bullet 4 \mathrm{Cl}$ has a fixed orientation and position, and does not shuttle along the dumbbell rapidly on the ${ }^{1} \mathrm{H}$ NMR timescale.

Photophysical studies. The UV/Vis absorption spectrum

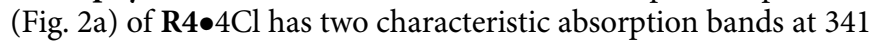
and $443 \mathrm{~nm}$, which can be attributed to electronic transitions in the pyrenyl and DAPP units, respectively. Despite the presence of the $\mathrm{CB} 6$ and $\gamma$-CD rings, $\mathbf{R} \mathbf{4} \bullet 4 \mathrm{Cl}$ forms aggregates $\left(\mathbf{R} \mathbf{4}^{\mathbf{4}}{ }_{\mathbf{a g g}}\right)$ in water, as confirmed (Supplementary Fig. 22) by dynamic light scattering experiments. A blue shift $(7 \mathrm{~nm})$ of the absorption band near $450 \mathrm{~nm}$ is recorded (Fig. 2b) in the concentration-dependent $\mathrm{UV} / \mathrm{V}$ is absorption spectrum of $\mathbf{R} \mathbf{4} \bullet \mathbf{C l}$ on dilution from 500 to $25 \mu \mathrm{M}$, while the shoulder evident at around $350 \mathrm{~nm}$, arising from the pyrene stopper, diminishes. The isosbestic points observed (Fig. 2b) at 431 and $492 \mathrm{~nm}$ suggest $\mathrm{t}^{34,35}$ that the aggregation is homogenous and non-cooperative ${ }^{36-38}$. By fitting the data to a dimerisation $\operatorname{model}^{39,40}$, the aggregation constant $K_{\text {agg }}$ was determined (Supplementary Figs $23-25$ ) to be $1.4 \times 10^{4} \mathrm{M}^{-1}$,

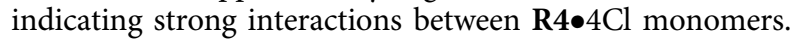

Irradiating a dilute solution $(5 \mu \mathrm{M})$ of $\mathbf{R} 4 \bullet 4 \mathrm{Cl}$ at excitation wavelengths of either 340 or $443 \mathrm{~nm}$ results in identical fluorescence emission spectra (Supplementary Fig. 26), with an emission maximum $(\Phi=52.4 \%$, Supplementary Table 2 and Supplementary Figs $27-44)$ at $510 \mathrm{~nm}$. No emission is observed at $390 \mathrm{~nm}$, indicating (Fig. 2a and Supplementary Fig. S41) the transfer of the excited state energy from pyrene to DAPP by a Förster resonance energy transfer (FRET) mechanism with nearquantitative ( $>99 \%$ ) efficiency. This remarkable FRET efficiency can be rationalised by considering the geometric constraints enforced by the rings that impart (i) a close-to-ideal spatial separation $(\sim 1.2 \AA$, calculated by molecular mechanics, Supplementary Figs $45-47$ ) between the FRET donors (pyrene) and acceptor (DAPP) in $\mathbf{R} 4 \bullet 4 \mathrm{Cl}$, while (ii) limiting conformational flexibility and (iii) preventing aggregation-induced quenching. These observations suggest that cooperative capture strategies 
a
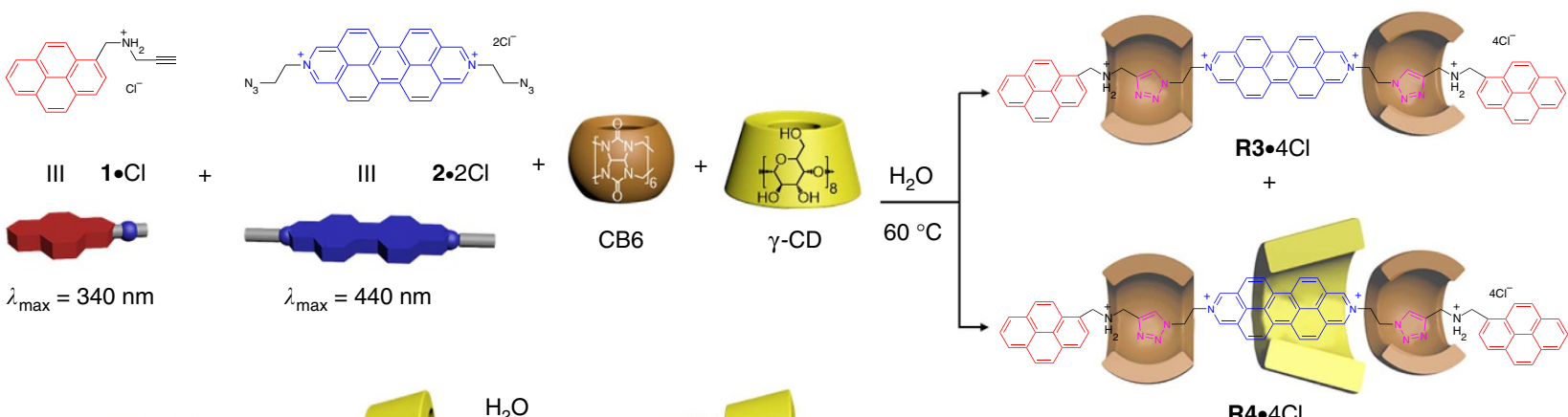

b
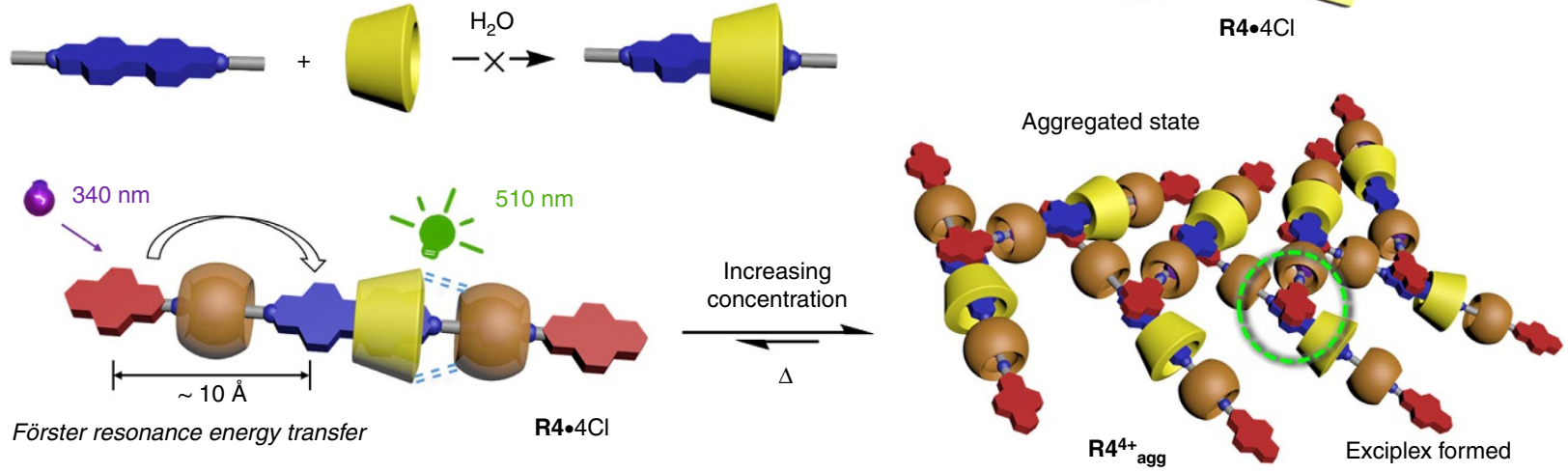

Förster resonance energy transfer
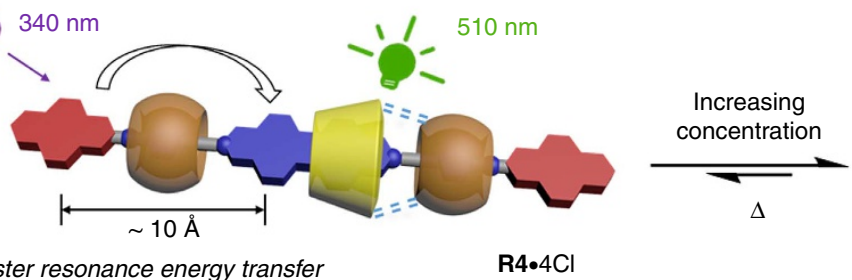

$\Delta$

d
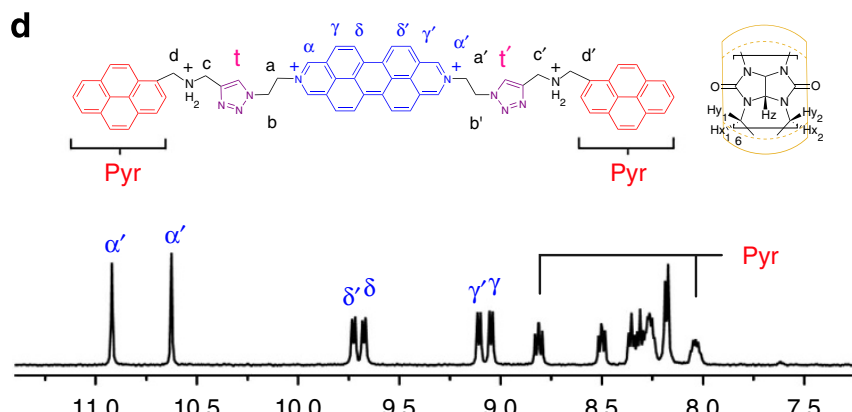

Pyr
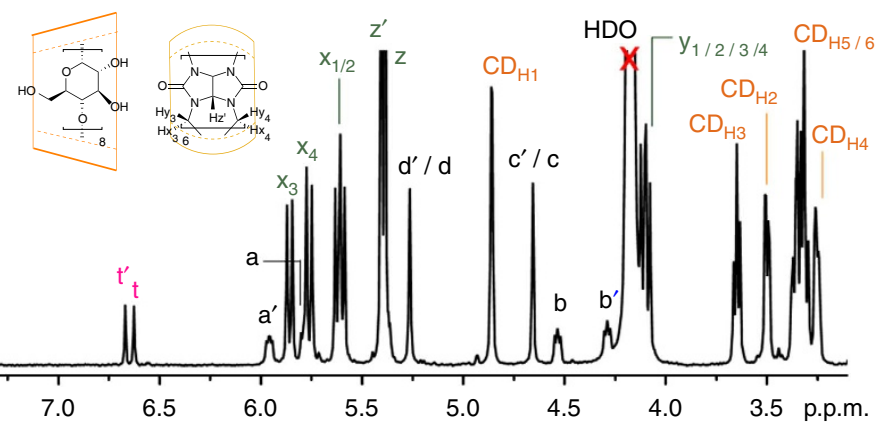

Figure 1 | Synthesis and characterization of heterorotaxanes. (a) Synthesis of the heterorotaxanes $\mathbf{R 3} \bullet 4 \mathrm{Cl}$ and $\mathbf{R 4 \bullet} 4 \mathrm{Cl}$ from the stopper $\mathbf{1 \bullet} \mathrm{Cl}$, the dumbbell precursor $\mathbf{2} \cdot 2 \mathrm{Cl}, \mathrm{CB} 6$ and $\gamma$-CD. (b) No complexation was observed between $\mathbf{2} \cdot 2 \mathrm{Cl}$ and $\gamma$-CD. (c) Graphical representation of the aggregation of $\mathbf{R 4}^{4+}$ monomers in response to changes in concentration or temperature. (d) ${ }^{1} \mathrm{H}$ NMR spectrum $(600 \mathrm{MHz})$ of $\mathbf{R 4 \bullet} 4 \mathrm{Cl}(1 \mathrm{mM})$ recorded in $\mathrm{D}_{2} \mathrm{O}$ at $80^{\circ} \mathrm{C}$.

have the potential to control the distances between fluorophores for applications such as bio-sensing 41,42 .

As R4•4Cl undergoes increased aggregation at higher and higher concentrations, its narrow emission band (Fig. 2c) at $510 \mathrm{~nm}$ is gradually replaced by a broad, featureless band around $610 \mathrm{~nm}$, implying that either excimers (DAPP homodimers) or exciplexes (pyrenyl-DAPP heterodimers) are being formed in the excited state. Circular dichroism spectra reveal (Fig. 2d) that the aggregation of $\mathbf{R 4 \bullet 4 C l}(200 \mu \mathrm{M})$ is temperature dependent. As the temperature is lowered from $80^{\circ} \mathrm{C}$ (monomeric state) to $2^{\circ} \mathrm{C}$ (aggregated state), the positive induced circular dichroism (ICD) signals diminish, as a negative ICD peak ${ }^{43-45}$ attributable to the pyrene stoppers appears at around $350 \mathrm{~nm}$, indicating that these stoppers congregate near the rims of the $\gamma-\mathrm{CD}$ ring. As $\gamma-\mathrm{CD}$ encircles DAPP, it follows that pyrene-DAPP heterodimers (Figs $1 \mathrm{c}$ and 2d), and their corresponding exciplexes, are responsible for the observed aggregation and emission behaviour of $\mathbf{R} 4 \bullet 4 \mathrm{Cl}$.

Dynamic supramolecular equilibria of the hetero[4]rotaxane. In aqueous solution, the disassembly (Fig. 3a) of aggregated heterorotaxanes $\left(\mathbf{R 4}^{\mathbf{4}}{ }_{\text {agg }}\right)$ is promoted by introducing $\gamma$-CD, which encircles the pyrene moieties of $\mathbf{R 4} \bullet \mathbf{4 C l}$ and prevents aggregation. The stepwise encapsulation process, which occurs via the formation of a $\mathbf{R 4}^{\mathbf{4}+} \subset \mathrm{CD}$ intermediate, favours a monomeric $\mathbf{R 4}^{\mathbf{4}+} \subset \mathrm{CD}_{2}$ complex in the presence of excess of $\gamma-\mathrm{CD}$, with an averaged equilibrium constant $K_{\mathrm{CD}}=\left(K_{1} \cdot K_{2}\right)^{1 / 2}$. As a result of the complex equilibria in solution, it is not easy to measure $K_{\mathrm{CD}}$ directly. To obtain a good estimate of the binding affinities between the pyrene moieties of $\mathbf{R} 4 \bullet 4 \mathrm{Cl}$ and $\gamma-\mathrm{CD}$, a reference heterorotaxane $\mathbf{S R 4} \mathbf{4} \mathrm{Cl}$ was synthesised and the averaged binding affinity $\left(0.9 \times 10^{4} \mathrm{M}^{-1}\right)$ between its pyrene moiety and $\gamma$-CD was measured (Supplementary Figs $48-55$ ) in $\mathrm{D}_{2} \mathrm{O}$. This encapsulation process can be reversed (Fig. 3a) by introducing a competitive binding agent (CBA), which competes for $\gamma$-CD in solution with an association constant, $K_{\mathbf{C B A}}$. As the aggregation constant $K_{a g g}$ and the encapsulation constant $K_{\mathrm{CD}}$ are of the same order of magnitude, addition of even a weak CBA, for example, 2-adamantylamine hydrochloride (Ad $\bullet \mathrm{Cl}, K_{\mathbf{C B A}}=$ $90 \mathrm{M}^{-1}$, Supplementary Figs $56-59$ ), will efficiently perturb the equilibria between $\mathbf{R 4}^{\mathbf{4}+}{ }_{\text {agg }}, \mathbf{R 4}^{\mathbf{4}+} \subset \mathrm{CD}_{2}$ and $\mathbf{R 4}^{\mathbf{4}+}$.

The dynamic nature of $\mathbf{R 4}^{\mathbf{4}}$ in aqueous solution affords us the opportunity to customize an emission profile that remains preserved in the solid state after removal of the solvent. The fluorescent emission spectrum (Fig. $3 b$ and Supplementary 

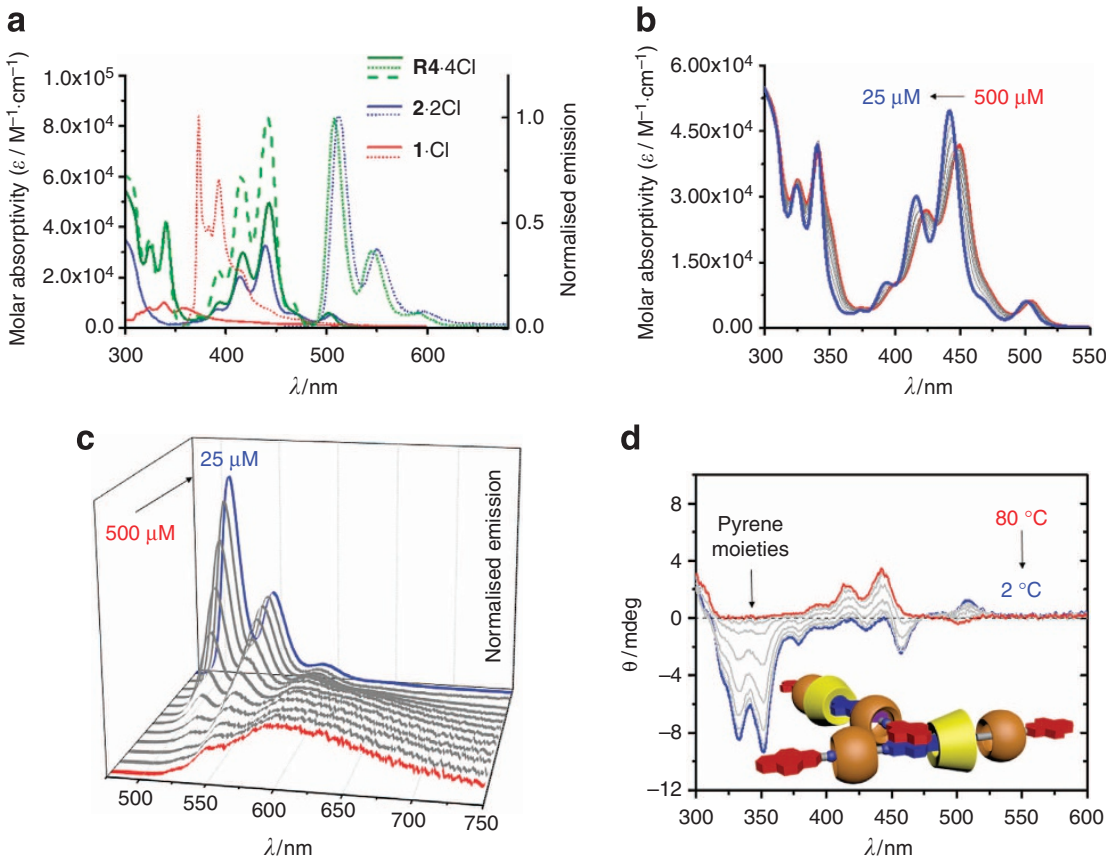

d

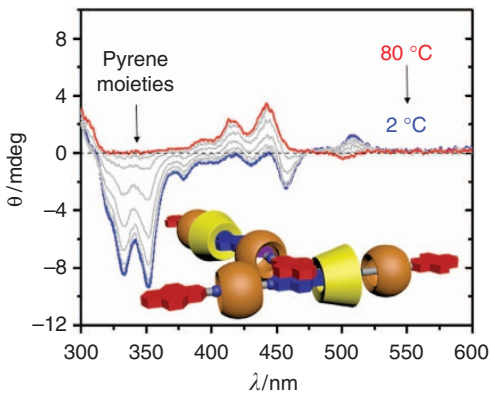

Figure 2 | Photophysical studies of $\mathbf{R 4 . 4 C l}$. (a) UV/Vis absorption (solid lines) and normalised fluorescence spectra (excitation: dashed lines, emission: dotted lines) of aqueous solutions of $\mathbf{R 4} \cdot 4 \mathrm{Cl}$ (green), stopper $\mathbf{1 \cdot} \mathrm{Cl}$ (red) and dumbbell precursor $\mathbf{2} \cdot 2 \mathrm{Cl}$ (blue). (b) Concentration-dependent $(25-500 \mu \mathrm{M}) \mathrm{UV} / \mathrm{Vis}$ absorption spectra of $\mathbf{R 4} \cdot 4 \mathrm{Cl}$ at $25^{\circ} \mathrm{C}$ in water. (c) Normalised concentration-dependent $(25-500 \mu \mathrm{M})$ fluorescence emission spectra $\left(\lambda_{\text {excitation }}=341 \mathrm{~nm}\right)$ of $\mathbf{R 4 \bullet} 4 \mathrm{Cl}$ at $25^{\circ} \mathrm{C}$ in water. (d) Temperature-dependent $\left(2-80^{\circ} \mathrm{C}\right)$ ICD spectra $(200 \mu \mathrm{M})$ of $\mathbf{R 4 \bullet 4 C l}$ in water.

a

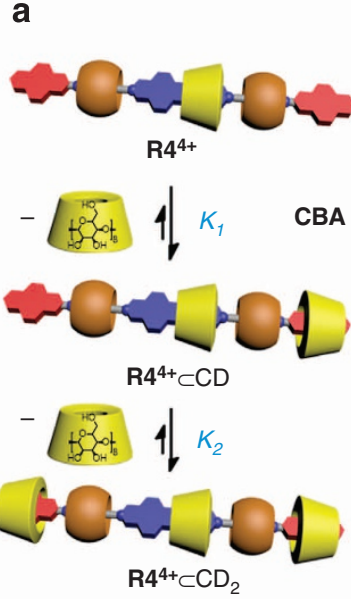

$K_{C D}=\left(K_{1} \cdot K_{2}\right)^{1 / 2}=0.9 \times 10^{4} \mathrm{M}^{-1}$
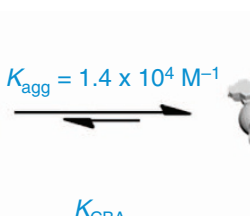

$\mathrm{CD} \stackrel{K_{\mathrm{CBA}}}{\longrightarrow}$ CBA $\subset \mathrm{CD}$
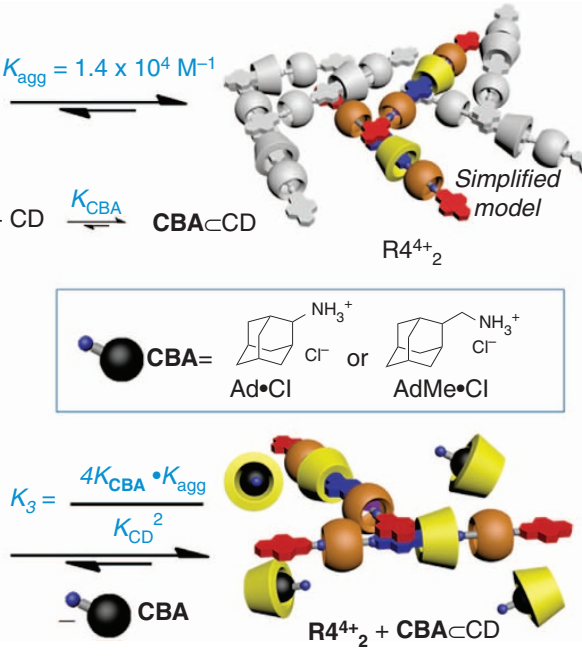

b

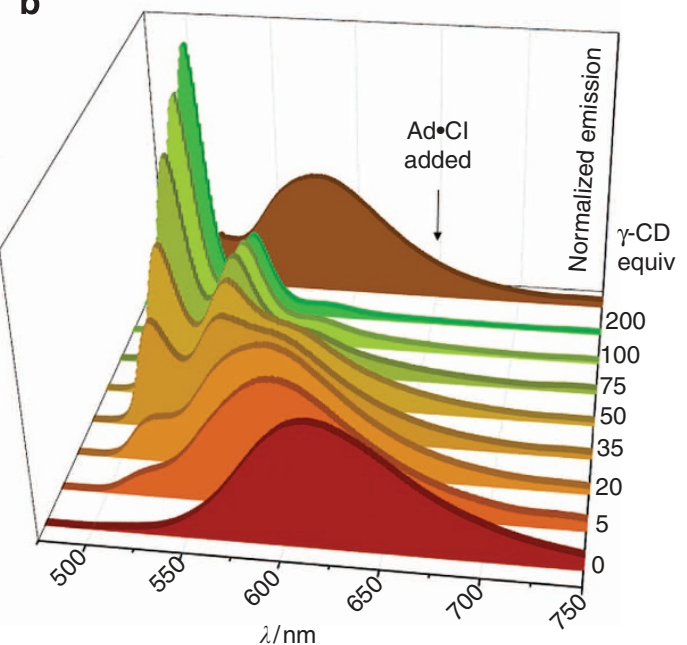

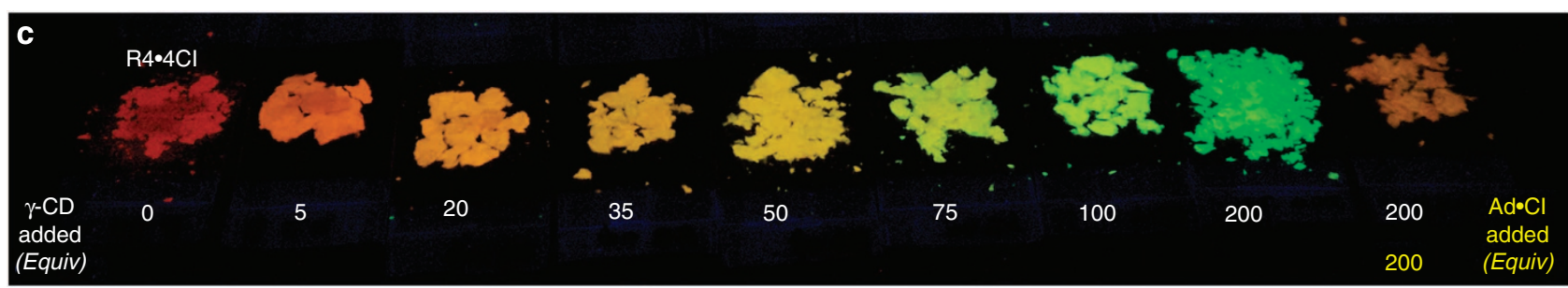

Figure 3 | Equilibrium network and solid-state fluorescence studies. (a) Graphical representation of the equilibria involving $\mathbf{R 4}^{\mathbf{4}+}$ as its $\mathrm{Cl}^{-}$salt in the presence of $\gamma$-CD and CBAs. (b) Solid-state fluorescence spectra ( $\lambda_{\text {excitation }}=347 \mathrm{~nm}$ ) of $\mathbf{R 4 \cdot 4 C l}$ on adding $0-200$ equiv. of $\gamma$-CD, followed by 200 equiv. of $\mathrm{Ad} \bullet \mathbf{C l}$. (c) Powders obtained from homogeneous mixtures of $\mathbf{R 4 \bullet 4 C l}$ and varying amounts (0-200 equiv) of $\gamma$-CD and $\mathrm{Ad} \bullet \mathbf{C l}(200$ equiv) under UV light.

Figs $60-64)$ of the amorphous $\mathbf{R 4}^{\mathbf{4}+}$ agg $\left(\lambda_{\max }=610 \mathrm{~nm}\right.$, $\Phi=7.7 \%$ ) is very similar to its emission (Fig. 2c) in a concentrated aqueous solution (Supplementary Figs 65-66).
On the addition of $\gamma-\mathrm{CD}$, the solid-state fluorescent emission spectra become gradually blue-shifted to $510 \mathrm{~nm}(\Phi=42.5 \%)$, with the emission colour (Fig. 3c) changing from red to green. 
Solid-state emission is also conserved from solution in the presence of a CBA. For example, the addition of 200 equiv. of $\mathrm{Ad} \bullet \mathrm{Cl}$ to a mixture comprising $\mathbf{R} 4 \bullet 4 \mathrm{Cl}: \gamma-\mathrm{CD}$ (molar ratio: $1: 200$ ) results in a red-shift (Fig. 3b,c) of the emission back to $\lambda_{\max }=580$ $\mathrm{nm}$. Thus, by changing the ratio of $\mathbf{R 4} \bullet 4 \mathrm{Cl}, \gamma-\mathrm{CD}$ and $\mathrm{Ad} \bullet \mathrm{Cl}$, the solid-state fluorescence of the material can be tuned reversibly over a wide range of colours from green through to red. This stimulus-responsive tuning of fluorescence spectra in the solid state, over such a wide range of colours and under ambient conditions, is unique and holds promise for applications in security printing technology.

Applications as fluorescent inks. By loading $\mathbf{R} 4^{4+}$-based aqueous solutions (inks) into fountain pens, information can be written (Fig. 4), which is then revealed under UV light. By applying colourless $\gamma$-CD and Ad•Cl inks on top of the fluorescent $\mathbf{R} 4 \bullet 4 \mathrm{Cl}$ ink (Fig. 4a), additional information can be added $\left(\mathbf{R 4}^{\mathbf{4}+}{ }_{\mathbf{a g g}} \rightarrow \mathbf{R} 4^{\mathbf{4}+} \subset \mathrm{CD}_{2}\right)$ or erased $\left(\mathbf{R 4}^{\mathbf{4}+} \subset \mathrm{CD}_{2} \rightarrow\right.$ $\left.\mathbf{R} \mathbf{4}^{\mathbf{4}}{ }_{\mathbf{a g g}}\right)$ on pre-existing images that is only noticeable (Supplementary Fig. 67 and Supplementary Movie 1) under UV light. During handwriting experiments, we found that the $\mathbf{R} \mathbf{4}^{\mathbf{4}} \subset \subset \mathrm{CD}_{2}$ ink exhibits an unusual phenomenon, that is, the colour of its emission depends (Fig. 4b) on the type of paper (Supplementary Movie 2). For example, on rag paper, newsprint and banknotes, $\mathbf{R} 4 \bullet 4 \mathrm{Cl}$ appears reddish-orange and $\mathbf{R} \mathbf{4}^{\mathbf{4}}+\subset \mathrm{CD}_{2}$ appears green (Fig. 4b) under UV light, which is consistent (Fig. $3 \mathrm{c}$ ) with the corresponding powders. On different kinds of ordinary white paper (Fig. 4b, Supplementary Figs $68-70$ and Supplementary Table 3), however, both of a

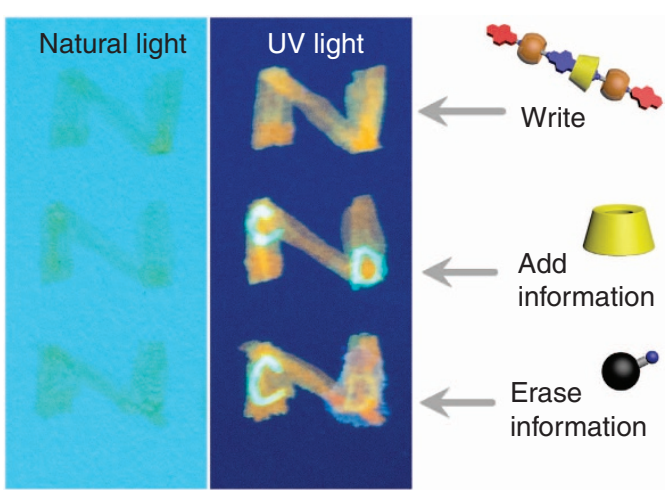

b

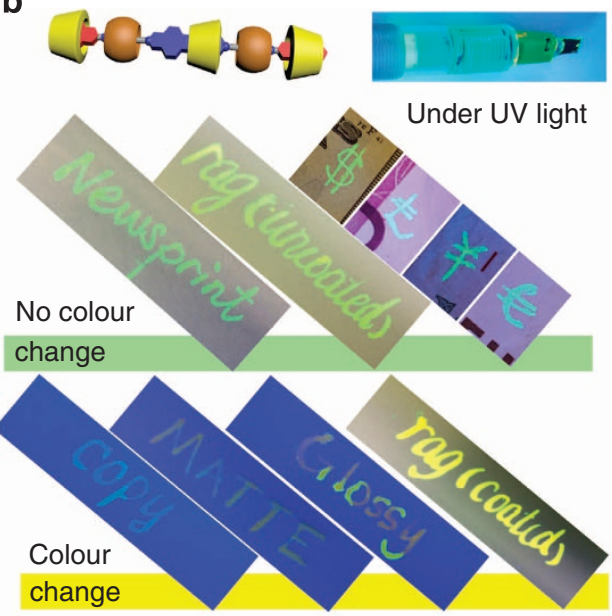

C
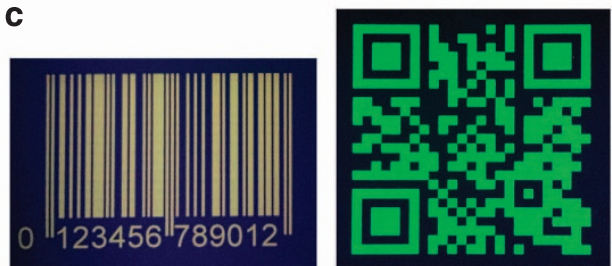

d

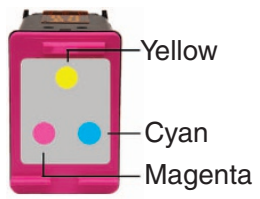

Tri-colour ink cartridge

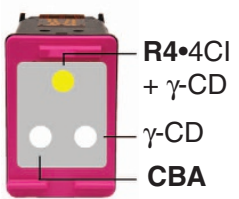

Customised ink cartridge

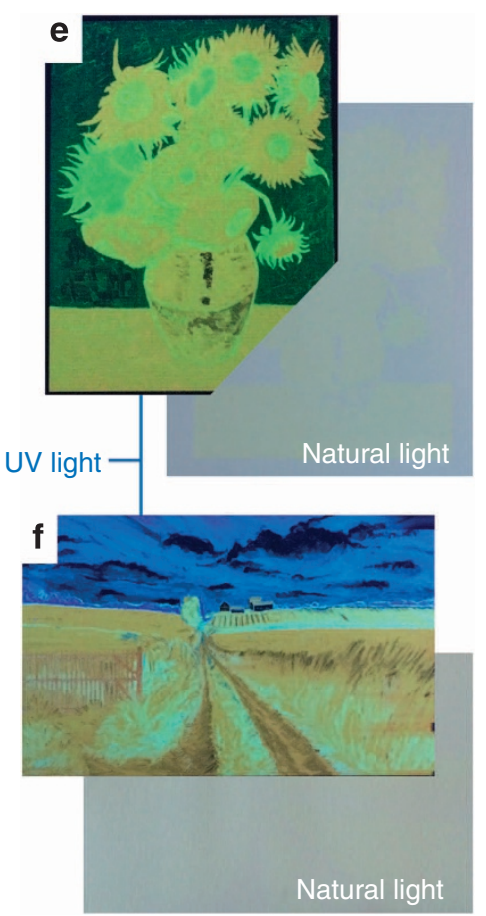

Figure 4 | Security features of the heterorotaxane $\mathbf{R 4}^{\mathbf{4}+}$ - and its complex $\mathbf{R 4}^{\mathbf{4}}+\subset \mathbf{C D}_{\mathbf{2}}$-based fluorescent inks. (a) Reversibly adding and erasing information on the fluorescent ink with $\gamma$-CD and $\mathrm{Ad} \bullet \mathrm{Cl}$ aqueous solution. (b) Surface-dependent fluorescence of $\mathbf{R 4}^{\mathbf{4}+} \subset \mathrm{CD}_{2}$ ink on different paper media (newsprint, coated and uncoated rag paper, banknotes, copy, matte and glossy white paper) under UV light. (c) A UV barcode and a QR code under UV light printed using a customized black inkjet cartridge filled with $\mathbf{R 4}^{\mathbf{4}+}$ and $\mathbf{R 4}^{\mathbf{4}}+\subset \mathrm{CD}_{2}$ ink, respectively. (d) Graphical representations of a customized tri-colour inkjet cartridge, in which aqueous solutions of $\mathbf{R 4 \bullet} 4 \mathrm{Cl} / \gamma-\mathrm{CD}(\mathbf{R 4 \bullet 4 C l : ~} 1 \mathrm{mM}, \gamma-\mathrm{CD}: 200 \mathrm{mM})$, a CBA and $\gamma$-CD occupy the yellow, magenta and cyan colour channels, respectively. (e) Fluorescent replica of van Gogh's 'Sunflowers' on rag paper printed using the customized tri-colour inkjet cartridge under UV and natural light. (f) Fluorescent image printed using an inkjet cartridge under UV and natural light, in which the cyan channel was loaded with $\gamma$-CD and PyMe $\bullet \mathrm{Cl}$. 
these inks appear reddish-orange. The change of colour is most likely a result of noncovalent bonding interactions with papers of different compositions.

$\mathbf{R 4}^{\mathbf{4}}{ }^{+}$-based inks are also compatible with inkjet printing technology. A monochromic barcode and a QR code printed (Fig. 4c) on paper from an inkjet cartridge contains information that, although invisible under natural light, can be read (Supplementary Figs $71-72$ and Supplementary Movie 3) on a smartphone under UV light. The supramolecular encapsulation/ competition between $\mathbf{R} \mathbf{4}^{\mathbf{4}}, \gamma-\mathrm{CD}$ and $\mathrm{Ad} \bullet \mathrm{Cl}$ is established rapidly (milliseconds) before the inks dry during the printing process, making it possible to print polychromic fluorescent images. By loading aqueous solutions of $\mathbf{R 4}^{\mathbf{4}+} \subset \mathrm{CD}_{2}\left(\mathbf{R 4}^{\mathbf{4}}\right.$ : $\gamma-\mathrm{CD}=1: 50), \gamma-\mathrm{CD}$ and $\mathrm{Ad} \bullet \mathrm{Cl}$ into a tri-colour inkjet cartridge (Fig. 4d and Supplementary Fig. 73), we have printed a fluorescent reproduction (Fig. 4e) of van Gogh's 'Sunflowers' with good colour resolution. The colour range of the fluorescent inks can be expanded to accommodate RGB printing (Fig. $4 \mathrm{f}$ and Supplementary Figs 74-76) by choosing a fluorescent CBA with blue emission, such as the terminal fragment of $\mathbf{R} 4 \bullet 4 \mathrm{Cl}$, 1-pyrenemethylamine hydrochloride $(\mathrm{PyMe} \bullet \mathrm{Cl})$. It is worth noting that, by reducing the amount of $\mathbf{R} 4 \bullet 4 \mathrm{Cl}$ applied to the paper, the images produced are invisible to the naked eye (Fig. 4f, right) under natural light.

Supramolecular encryption and authentication theory. At a fundamental level, the $\mathbf{R 4}^{\mathbf{4}+}$-based fluorescent inks provide an extensive fluorescent colour matrix for encryption coding. More importantly, the nonlinear dependence of this system's output on the concentrations of components and their equilibrium constants (Fig. 3a) points towards a general concept whereby complex supramolecular equilibria in aqueous solutions can be used as a chemical encryption method (Fig. 5). In principle, the colour of a dot printed by the customized tri-colour ink cartridge reflects a complex supramolecular equilibrium in the solution state, which can be simplified as

$$
\begin{aligned}
3 \mathrm{R}^{4+}+3 \mathrm{CD}+\mathrm{CBA} & \stackrel{4 K_{\mathrm{agg}} \bullet \mathrm{K}_{\mathrm{CD}}^{2} \bullet K_{\mathrm{CBA}}}{=} \mathrm{R}_{2}^{4+}+\mathrm{R}^{4+} \subset \mathrm{CD}_{2} \\
& +\mathrm{CBA} \subset \mathrm{CD}
\end{aligned}
$$

Since $K_{\text {agg }}$ and $K_{\mathrm{CD}}$ are fixed, three key parameters control the supramolecular equilibria and the subsequent fluorescent colour (Fig. 5 and Supplementary Fig. 77) under UV light for a given dot after printing: (1) $\left[\mathbf{R} \mathbf{4}^{\mathbf{4}}\right]_{0}$, reflecting the absolute amount of
$\mathbf{R} \mathbf{4}^{4+}$ ink applied on paper, (2) $[\mathrm{CD}]_{0}$ and $[\mathbf{C B A}]_{0}$, reflecting the ratio of $\mathbf{R 4}^{\mathbf{4}}$ agg and $\mathbf{R 4}^{\mathbf{4}+} \subset \mathrm{CD}_{2}$ on paper and (3) the chemical composition of $\mathbf{C B A}$, reflecting a different $K_{\mathbf{C B A}}$ in the supramolecular equilibrium. These variables constitute the encryption settings, which can be defined by the user in charge of security printing. By simply varying (i) the sequence (Fig. 5) of inks in Channels (1), (2), (3), (ii) the chemical composition of the CBA and (iii) the concentration of the inks, it is possible to generate a large number of fluorescent colour combinations. It is also worth noting that, a combination of different CBAs could be loaded in channels (2) and (3) simultaneously, thus introducing even more variables to the supramolecular equilibria. In this manner, it would be challenging for counterfeiters to reproduce a

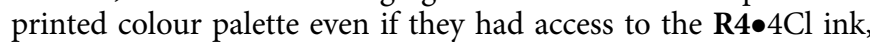
as they would also require a complete knowledge of (i) the CBA, of which there could be a large number of possibilities, as well as (ii) the paper media, (iii) channel assignments and (iv) initial ink concentrations. Even relatively small errors in initial concentrations can lead to obvious differences in the colour palette, by a margin which cannot be easily reverse engineered on account of the non-linearity of the equilibrium equations,

$$
\begin{aligned}
& I_{\lambda}=F_{\mathrm{R} 4^{4+}}^{\circ}+F^{\circ}{ }^{\circ} 4^{4+}{ }_{\mathrm{CD}} \bullet K_{\mathrm{CD}}\left([\mathrm{CD}]_{0}-3 m\right) \\
& +F^{\circ}{ }_{\mathrm{R}^{4+}} \mathrm{CCD}_{2} \bullet K_{\mathrm{CD}}^{2} \bullet\left([\mathrm{CD}]_{0}-3 m\right)^{2} \bullet\left(\left[\mathrm{R}^{4+}\right]_{0}-3 m\right) \\
& +F_{\mathrm{R} 42}^{\circ+} \bullet 4 K_{\mathrm{agg}} \bullet\left(\left[\mathrm{R} 4^{4+}\right]_{0}-3 m\right)^{2} \\
& +F^{\circ}{ }_{\mathrm{CBA}} \bullet\left([\mathrm{CBA}]_{0}-m\right)+F^{\circ} \mathrm{CBA} \subset \mathrm{CD} \bullet m
\end{aligned}
$$

where the fluorescence intensity $I_{\lambda}$ at a given wavelength $\lambda$ is the sum of the emission intensities of each component in an aqueous solution containing R4•4Cl, $\gamma$-CD and CBA. $F^{\circ}$ is the molar fluorescence coefficient at wavelength $\lambda$, and $m$ is the molar concentration of CBA being encapsulated by $\gamma-\mathrm{CD}$. See Supplementary Discussion for detailed derivations.

The dynamic nature of the inks also makes them amenable to a variety of fraud detection mechanisms. In principle, applying a layer of authentication agent(s) can re-establish the supramolecular equilibria in solution and shift the colour outputs (Fig. 5) on paper non-linearly, as described by equations (1) and (2). As the colour-changing process is dynamic and depends on the amount of the authentication agent(s) that has been applied, it is close to impossible that the dynamic colour-changing process could be precisely mimicked. Apart from applying authentication agent(s),

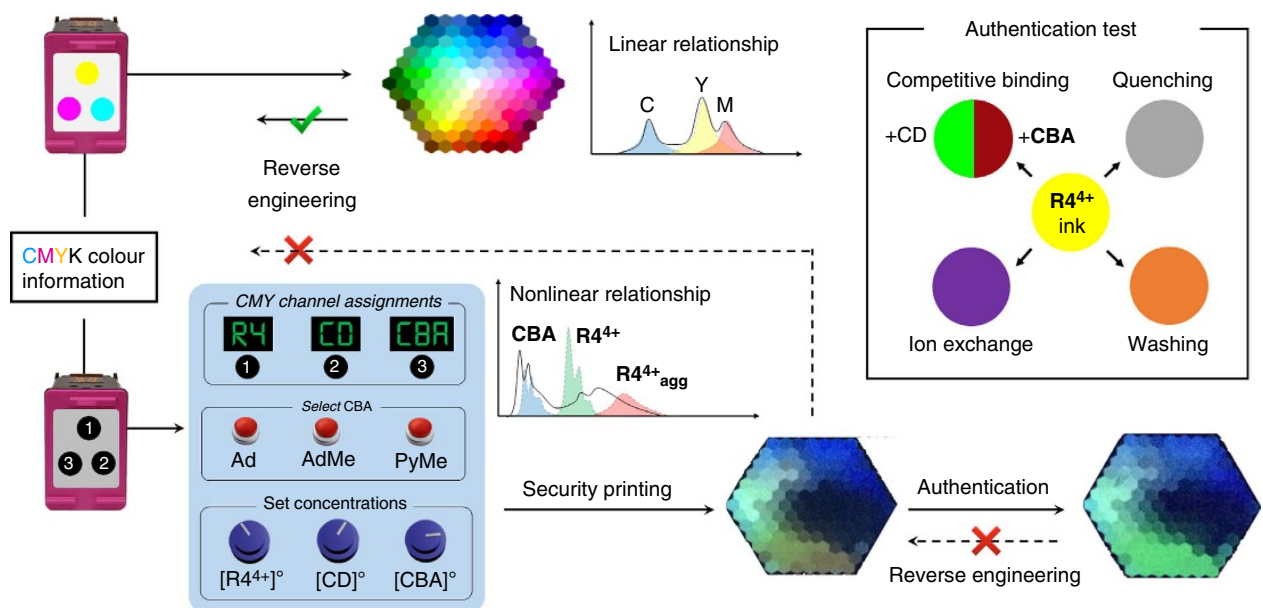

Figure 5 | Supramolecular encryption and fraud detection using the heterorotaxane-based fluorescent security inks. A comparison between conventional cyan-magenta-yellow-black (CMYK) printing and supramolecular encrypted printing technology. Inset: possible mechanisms to verify the authenticity of the protected colour document. 
which could induce a supramolecular equilibrium shift, mechanisms such as counterion exchange and fluorescence quenching can also be utilized (Fig. 5 inset) to change emission colours in real time.

Demonstration of the supramolecular encryption theory. In an attempt to demonstrate the non-linear nature of this system, we have printed fluorescent colour palettes (Fig. $6 \mathrm{a}-\mathrm{f}$ ) using various ink concentrations and different CBAs. A broad range of colours from green to red can be printed (Fig. 6b) when $\mathrm{Ad} \bullet \mathrm{Cl}$ is used as the competitor. The colour palette is sensitive to the association strength of the competitor as predicted and evidenced by the differences between images printed from equally concentrated solutions of $\mathrm{Ad} \bullet \mathrm{Cl}$ (Fig. 6b) and a stronger-binding competitor (Fig. 6c), namely, 1-adamantanemethylamine hydrochloride (AdMe $\bullet C l, K_{\mathrm{AdMe}}=127 \mathrm{M}^{-1}$, Supplementary Figs $58-59$ ). Reducing the concentrations of either CBA (Fig. 6c,d) or $\gamma$-CD (Fig. 6c,e) redistributes the colour spectrum in the yellowish-red region or greenish yellow region, respectively. The colour spectrum is expanded (Fig. 6f) by choosing a blue fluorescent CBA, for example, PyMe•Cl. The ability to exchange fluorescent and non-fluorescent CBAs in a modular and user-controlled manner elevates the anti-counterfeiting features possessed intrinsically by this new security ink.

Demonstration of authentication methods. Authentication mechanisms have also been demonstrated. For example, exposure of an encrypted image (Fig. 6g, centre) to aqueous solutions of non-fluorescent $\mathrm{AdMe} \bullet \mathrm{Cl}$ or $\gamma$-CD changes the existing colour gradient of the image, while printing fluorescent $\mathrm{PyMe} \bullet \mathrm{Cl}$ aqueous solution creates new colours, such as blue and purple by shifting the complex equilibria. Printing 1,3,6,8-pyrenetetrasulfonic acid tetrasodium $(\mathrm{PTSA} \bullet 4 \mathrm{Na})$ solution gives rise to new colours (Fig. 6g) as a result of counterion exchange. A characteristic colour change can also be brought about through the application of a quencher, such as tryptophan, or even by simply soaking the printed image in water for as little as $1-2 \mathrm{~min}$, during which time $\gamma-\mathrm{CD}$ and the CBA are washed away to some extent, thus shifting the equilibria. These authentication agents can discriminate, not only between images produced by the $\mathbf{R} \mathbf{4}^{\mathbf{4}+}$-based ink and other fluorescent dyes, but also between images produced using different $\mathbf{R} \mathbf{4}^{\mathbf{4}+} / \mathbf{C B A} / \gamma-\mathrm{CD}$ ink formulations. In a blind test, blocks of a given fluorescence colour (Fig. 6h, top), which appear almost identical to one another under UV light, but are formulated differently, were found to result in noticeably different colours (Fig. 6h bottom) after the application of the same amount of authentication agents. For further details, see Supplementary Fig. 78. As hundreds of chemicals meet the criteria to be CBAs, an extremely large library of different ink systems and authentication tests using this supramolecular encryption method can, in principle, be generated.

\section{Discussion}

In summary, we have developed a stimulus-responsive solid-state fluorescent heterorotaxane, which is easily prepared from simple
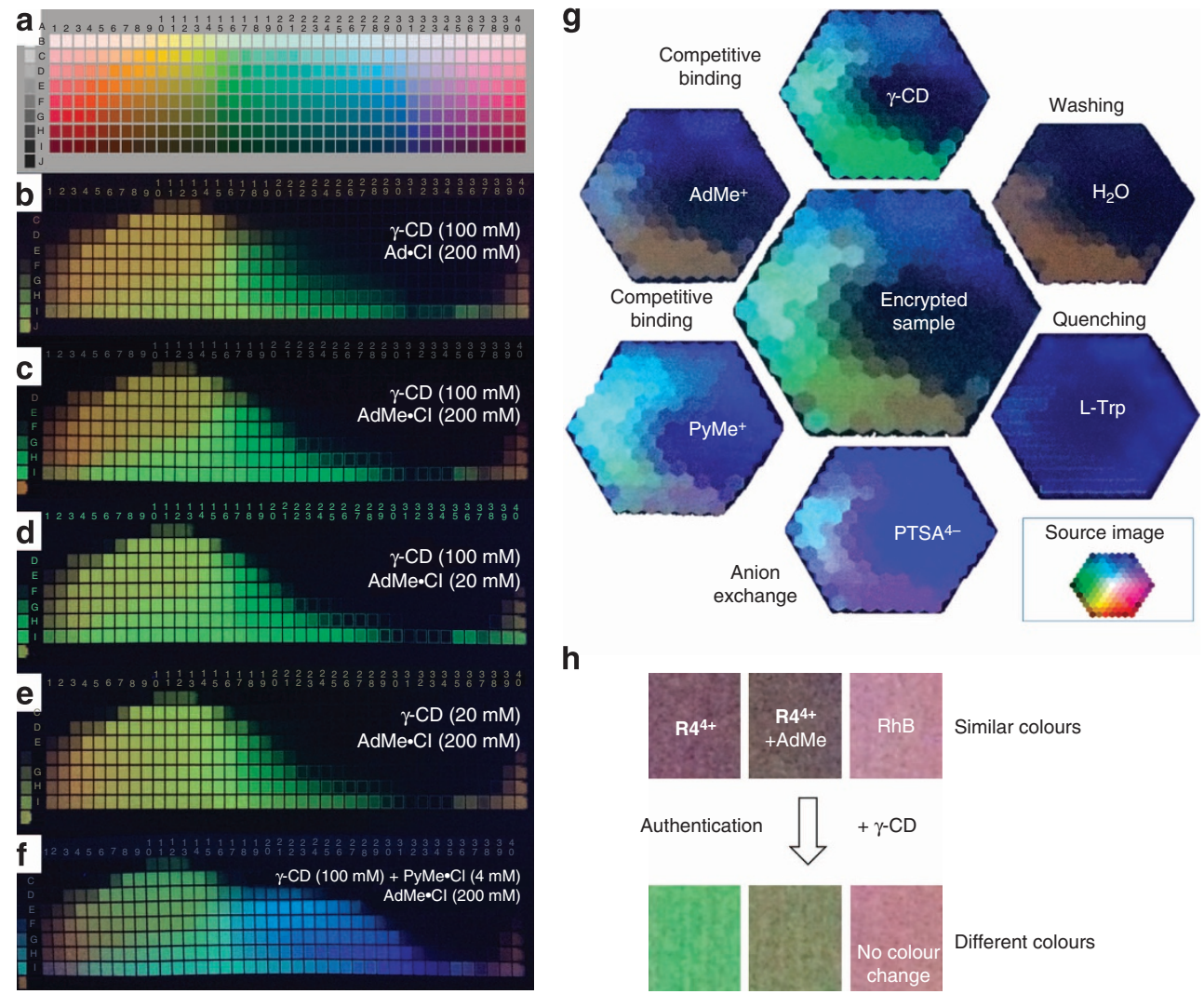

h

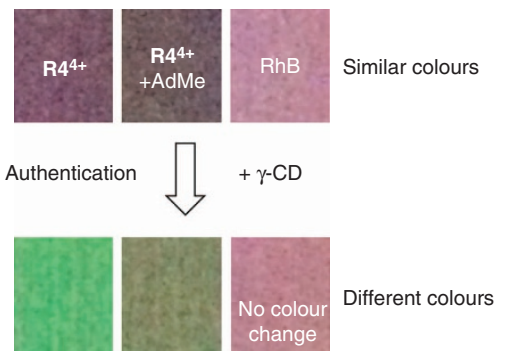

Figure 6 | Demonstration of the supramolecular encryption and authentication using the heterorotaxane-based fluorescent security inks.

(a) A standard colour palette. (b-f) Colour palette images produced using the customized tri-colour inkjet cartridge with (b) $\mathrm{Ad} \bullet \mathrm{Cl}(200 \mathrm{mM}$ ),

(c) AdMe•Cl $(200 \mathrm{mM})$ and $(\mathbf{d}) \mathrm{AdMe} \bullet \mathrm{Cl}(20 \mathrm{mM})$ in channel (3) (e) $\gamma-\mathrm{CD}(20 \mathrm{mM})$ and (f) $\gamma-\mathrm{CD}(100 \mathrm{mM})$ with PyMe $\bullet \mathrm{Cl}(4 \mathrm{mM})$ in channel (2), respectively. R4•4Cl $+\gamma-\mathrm{CD}(\mathbf{R 4} \bullet 4 \mathrm{Cl}$ : $1 \mathrm{mM}, \gamma-\mathrm{CD}: 40 \mathrm{mM})$ solution was loaded in channel (1) in the tri-colour inkjet cartridge. (g) Encrypted polychromic colour palette samples produced by the customized inkjet cartridge (centre) and its derivatives (around the periphery, after printing a layer of authentication reagents) under UV light. (h) Similar colours produced by $\mathbf{R 4}^{\mathbf{4}+}$-based security inks have composition-dependent response after chemical authentication. No distinguishable colour change is observed after chemical authentication when rhodamine B (RhB) is applied as the fluorescent ink. 
starting materials in high yield by a cooperative capture method. We have applied it as a component of fluorescent security inks with built-in supramolecular encryption. The inks are well-placed for assimilation into a commercial setting on account of the simple and high-yielding synthesis of the heterorotaxane from commodity chemicals. The solid-state emission of these security inks can be fine-tuned over a wide emission range $(\sim 100 \mathrm{~nm})$ with rapid response (milliseconds) to chemical stimuli. Printed information is encrypted in a chemical language based on a nonlinear equation that describes the dynamic equilibrium network. A potentially enormous library of different fluorescent colour combinations can be generated. In contrast with conventional dyes, the encrypted information printed using the heterorotaxane inks can be verified by chemical authentication methods without revealing the original colour image information. The interplay of fluorescence output with dynamic supramolecular equilibria that we observed quite fortuitously could be a general phenomenon that is not exclusive to the heterorotaxane, or even to mechanically interlocked molecules. It opens up a new way to encrypt and protect information in a manner that is far from easy to mimic.

\section{Methods}

Synthesis and characterization of the heterorotaxane $\mathbf{R 4 \bullet 4 C l}$. Stopper precursor $1 \bullet \mathrm{Cl}(67 \mathrm{mg}, 0.22 \mathrm{mmol})$, dumbbell precursor $2 \bullet 2 \mathrm{Cl}(54 \mathrm{mg}, 0.10 \mathrm{mmol})$ and $\gamma$-CD $(1287 \mathrm{mg}, 1.00 \mathrm{mmol})$ were mixed in $\mathrm{H}_{2} \mathrm{O}(35 \mathrm{ml})$ and stirred at $60^{\circ} \mathrm{C}$ for $10 \mathrm{~min}$ before CB6 $(250 \mathrm{mg}, 0.25 \mathrm{mmol})$ was added. The reaction mixture was stirred at $60^{\circ} \mathrm{C}$ for $3 \mathrm{~h}$. Insoluble residues were filtered off from the reaction mixture. The filtrate was loaded directly onto a reverse phase C18 column (150 gram, RediSep Rf Gold C18Aq) on an automatic column chromatographic system (Combiflash Rf200, Teledyne Isco) and chromatographed in $\mathrm{H}_{2} \mathrm{O} / \mathrm{MeCN} /$ $0.1 \%$ TFA with a gradient from 0 to $60 \% \mathrm{MeCN}$ over $40 \mathrm{~min}$ at a flow rate of $85 \mathrm{ml} \mathrm{min}^{-1}$. Fractions containing $\mathbf{R 4}^{\mathbf{4}}+$ were collected and the counterions of $\mathbf{R 4}^{\mathbf{4}+}$ were exchanged to $\mathrm{PF}_{6}^{-}$on addition of an excess of aqueous $\mathrm{NH}_{4} \mathrm{PF}_{6}$. The

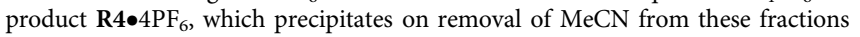
under reduced pressure, was collected by vacuum filtration and washed extensively with $\mathrm{H}_{2} \mathrm{O}$. The hetero[4] rotaxane $\mathbf{R} 4 \bullet 4 \mathrm{Cl}$ was obtained after a second counterion exchange by precipitation from an $\mathrm{MeCN}$ solution of $\mathbf{R} 4 \bullet 4 \mathrm{PF}_{6}$ with an excess of tetrabutylammonium chloride. The yellow precipitate was collected by vacuum filtration, washed with excess $\mathrm{MeCN}$ and dried under vacuum to afford $\mathbf{R 4 \bullet 4 C l}$ $(371 \mathrm{mg}, 83 \%)$ as an orange powder. ${ }^{1} \mathrm{H}$ NMR $\left(600 \mathrm{MHz}, \mathrm{D}_{2} \mathrm{O}, 353 \mathrm{~K}\right): \delta=10.92$ $(\mathrm{s}, 2 \mathrm{H}), 10.63(\mathrm{~s}, 2 \mathrm{H}), 9.73(\mathrm{~d}, J=9.5 \mathrm{~Hz}, 2 \mathrm{H}), 9.68(\mathrm{~d}, J=9.4 \mathrm{~Hz}, 2 \mathrm{H}), 9.11(\mathrm{~d}$, $J=9.3 \mathrm{~Hz}, 2 \mathrm{H}), 9.05(\mathrm{~d}, J=9.3 \mathrm{~Hz}, 2 \mathrm{H}), 8.81(\mathrm{dd}, J=12.4,9.3 \mathrm{~Hz}, 2 \mathrm{H}), 8.50(\mathrm{dd}$, $J=11.5,7.9 \mathrm{~Hz}, 2 \mathrm{H}), 8.40-8.22(\mathrm{~m}, 8 \mathrm{H}), 8.18(\mathrm{~d}, J=8.9 \mathrm{~Hz}, 4 \mathrm{H}), 8.08-7.98(\mathrm{~m}$, $2 \mathrm{H}), 6.67(\mathrm{~s}, 1 \mathrm{H}), 6.63(\mathrm{~s}, 1 \mathrm{H}), 5.96(\mathrm{t}, J=8.8 \mathrm{~Hz}, 2 \mathrm{H}), 5.86(\mathrm{~d}, J=15.3 \mathrm{~Hz}, 6 \mathrm{H})$, $5.80(\mathrm{~m}, 2 \mathrm{H}), 5.76(\mathrm{~d}, J=15.5 \mathrm{~Hz}, 6 \mathrm{H}), 5.62(\mathrm{~d}, J=15.5,6 \mathrm{H}), 5.60(\mathrm{~d}, J=15.5,6 \mathrm{H})$, $5.40(\mathrm{~s}, 12 \mathrm{H}), 5.39(\mathrm{~s}, 12 \mathrm{H}), 5.26(\mathrm{~s}, 4 \mathrm{H}), 4.86(\mathrm{~d}, J=3.8 \mathrm{~Hz}, 8 \mathrm{H}), 4.66(\mathrm{~s}, 4 \mathrm{H}), 4.53$ $(\mathrm{t}, J=9.0 \mathrm{~Hz}, 2 \mathrm{H}), 4.29(\mathrm{t}, J=8.9 \mathrm{~Hz}, 2 \mathrm{H}), 4.10(\mathrm{~m}, 24 \mathrm{H}), 3.65(\mathrm{t}, J=9.5 \mathrm{~Hz}, 8 \mathrm{H})$, 3.50 (dd, $J=10.0,3.8 \mathrm{~Hz}, 8 \mathrm{H}), 3.41-3.20$ (m, 32H). HR-ESI-MS: calcd for $[M-4 \mathrm{Cl}]^{4+} m / z=1,074.8630$, found $m / z=1,074.8623 ;[M-\mathrm{H}-4 \mathrm{Cl}]^{3+}$ $m / z=1,432.8149$, found $m / z=1,432.8123 ;[M-3 \mathrm{Cl}]^{3+} m / z=1,445.1406$, found $m / z=1,445.1369$.

Detailed synthesis and characterisation of stopper and rod precursors, rotaxanes $\mathbf{R} 3 \bullet 4 \mathrm{Cl}$ and SR4•4Cl are available in Supplementary Methods.

Photophysical studies of the heterorotaxane $\mathbf{R 4 \bullet 4 C l}$. The UV/Vis spectra of the sample solutions were measured on a Shimadzu UV/Vis/NIR spectrometer (UV 3600 model) with a cell temperature controller. Quartz cuvettes with 1 or $10 \mathrm{~mm}$ pathway were used to record the UV/Vis spectra. The fluorescence excitation and emission spectra of the sample solutions were recorded on a HORIBA fluorometer (fluoroMax-4 model). Circular dichroism spectra were recorded on a JASCO circular dichroism spectrophotometer (J-815 model) with a temperature controller. Solid-state UV/Vis spectra were recorded on a Perkin Elmer UV/Vis/NIR spectrometer (LAMBDA 1050 model) equipped with an integrating sphere. Solid-state fluorescence spectra were recorded on an ISS fluorometer ( $\mathrm{PCl}$ model) equipped with a variable-angle, front surface sample compartment.

Ink writing tests. Four types of inks for pen writing were prepared using $\mathbf{R} 4 \bullet 4 \mathrm{Cl}$ $(0.5 \mathrm{mM}), \mathrm{Ad} \bullet \mathrm{Cl}(100 \mathrm{mM}), \gamma-\mathrm{CD}(100 \mathrm{mM})$ and $\mathbf{R} 4 \subset \mathrm{CD}_{2}(\mathbf{R} 4 \bullet 4 \mathrm{Cl}=0.5 \mathrm{mM}, \gamma-$ $\mathrm{CD}=100 \mathrm{mM}$ ) solutions, respectively. Typically, $0.5 \mathrm{ml}$ of the ink was loaded in a fountain pen for writing tests. A wide selection of paper-based printing media has been tested, including copy papers (various brands and models), matte presentation paper (HP), glossy presentation paper (HP), resume paper (25 and $100 \%$ cotton), newsprint paper, rag paper (100\% cotton, without optical brightener) and cigarette rolling paper. Banknote identification tests were performed on genuine banknotes of US dollars, British pounds sterling, Euros, Chinese Yuan and Japanese Yen. In these tests, the corresponding currency symbols $(\$, £, €$ and $¥)$ were drawn on the testing banknotes using the fountain pen filled with $\mathbf{R 4} \subset \mathrm{CD}_{2}$ ink.

Ink printing tests. Printing tests were performed on an HP inkjet printer (Photosmart CP4780 model) and an HP colour laser printer (CP1025nw model) with customized ink cartridges and original toners, respectively. Rag paper $(100 \%$ cotton, without optical brightener, no surface coating side) was chosen for most printing tests based on the ink writing test results.

Ink cartridges for printing tests were customized from HP black and tri-colour cartridges (HP60 model). The filled inks were removed from the cartridge, which was washed extensively with $\mathrm{H}_{2} \mathrm{O}$ and $\mathrm{EtOH}$. Aqueous solutions of $\mathbf{R 4 \bullet 4 C l}(4 \mathrm{~mL}$ $0.5 \mathrm{mM})$ and $\mathbf{R} 4 \subset \mathrm{CD}_{2}(4 \mathrm{ml}, \mathbf{R} 4 \bullet 4 \mathrm{Cl}=0.5 \mathrm{mM}, \gamma-\mathrm{CD}=100 \mathrm{mM})$ were loaded in two empty, clean black ink cartridges, respectively, to perform the monochromic printing tests. In the polychromic printing tests, aqueous solutions of $\mathrm{Ad} \bullet \mathrm{Cl}(2 \mathrm{ml}$, $100 \mathrm{mM}), \mathbf{R} 4 / \gamma-\mathrm{CD}(2 \mathrm{ml}, \mathbf{R} 4 \bullet 4 \mathrm{Cl}=0.5 \mathrm{mM}, \gamma-\mathrm{CD}=25 \mathrm{mM})$ and $\gamma-\mathrm{CD}(2 \mathrm{ml}$, $100 \mathrm{mM}$ ) were loaded in the magenta, yellow and cyan channels of the cleaned tricolour ink cartridge, respectively. Fluorescent colour under UV light was tuned by controlling the proportion of the three inks in the customized tri-colour ink cartridge.

\section{References}

1. Hide, F. et al. Semiconducting polymers: a new class of solid-state laser materials. Science 273, 1833-1836 (1996)

2. Zhu, X. H., Peng, J. B., Caoa, Y. \& Roncali, J. Solution-processable singlematerial molecular emitters for organic light-emitting devices. Chem. Soc. Rev. 40, 3509-3524 (2011).

3. Santra, M. et al. Dramatic substituent effects on the photoluminescence of boron complexes of 2-(benzothiazol-2-yl)phenols. Chem. Eur. J. 18, 9886-9893 (2012).

4. Sasabe, H. et al. 3,3'-Bicarbazole-based host materials for high-efficiency blue phosphorescent OLEDs with extremely low driving voltage. Adv. Mater. 24, 3212-3217 (2012).

5. Kumar, K. et al. Printing colour at the optical diffraction limit. Nat. Nanotechnol. 7, 557-561 (2012).

6. Lu, Y. Q. et al. Tunable lifetime multiplexing using luminescent nanocrystals. Nat. Photonics 8, 33-37 (2014).

7. Deisingh, A. K. Pharmaceutical counterfeiting. Analyst 130, 271-279 (2005).

8. Yoon, B. et al. Recent functional material based approaches to prevent and detect counterfeiting. J. Mater. Chem. C 1, 2388-2403 (2013).

9. Prime, E. L. \& Solomon, D. H. Australia's plastic banknotes: fighting counterfeit currency. Angew. Chem. Int. Ed. 49, 3726-3736 (2010).

10. Kishimura, A., Yamashita, T., Yamaguchi, K. \& Aida, T. Rewritable phosphorescent paper by the control of competing kinetic and thermodynamic self-assembling events. Nat. Mater. 4, 546-549 (2005).

11. Mutai, T., Satou, H. \& Araki, K. Reproducible on-off switching of solid-state luminescence by controlling molecular packing through heat-mode interconversion. Nat. Mater. 4, 685-687 (2005)

12. Perruchas, S. et al. Mechanochromic and thermochromic luminescence of a copper iodide cluster. J. Am. Chem. Soc. 132, 10967-10969 (2010).

13. Yan, D. P. et al. Reversibly thermochromic, fluorescent ultrathin films with a supramolecular architecture. Angew. Chem. Int. Ed. 50, 720-723 (2011).

14. $\mathrm{Wu}, \mathrm{Y}$. et al. Quantitative photoswitching in bis(dithiazole)ethene enables modulation of light for encoding optical signals. Angew. Chem. Int. Ed. 53, 2090-2094 (2014).

15. Li, K. et al. Reversible photochromic system based on rhodamine B salicylaldehyde hydrazone metal complex. J. Am. Chem. Soc. 136, 1643-1649 (2014).

16. Tian, H. \& Yang, S. J. Recent progresses on diarylethene based photochromic switches. Chem. Soc. Rev. 33, 85-97 (2004).

17. Dong, Y. et al. Piezochromic luminescence based on the molecular aggregation of 9,10-bis((E)-2-(pyrid-2-yl)vinyl)anthracene. Angew. Chem. Int. Ed. 51, 10782-10785 (2012).

18. Sagara, Y. \& Kato, T. Mechanically induced luminescence changes in molecular assemblies. Nat. Chem. 1, 605-610 (2009).

19. Sagara, Y. \& Kato, T. Brightly tricolored mechanochromic luminescence from a single-luminophore liquid crystal: reversible writing and erasing of images. Angew. Chem. Int. Ed. 50, 9128-9132 (2011).

20. Dias, H. V. R., Diyabalanage, H. V. K., Rawashdeh-Omary, M. A., Franzman M. A. \& Omary, M. A. Bright phosphorescence of a trinuclear copper(I) complex: Luminescence thermochromism, solvatochromism, and concentration luminochromism. J. Am. Chem. Soc. 125, 12072-12073 (2003).

21. Liu, Y., Wang, K. R., Guo, D. S. \& Jiang, B. P. Supramolecular assembly of perylene bisimide with beta-cyclodextrin grafts as a solid-state fluorescence sensor for vapor detection. Adv. Funct. Mater. 19, 2230-2235 (2009). 
22. Yoon, S. J. et al. Multistimuli two-color luminescence switching via different slip-stacking of highly fluorescent molecular sheets. J. Am. Chem. Soc. 132, 13675-13683 (2010).

23. Ni, J., Zhang, X., Wu, Y. H., Zhang, L. Y. \& Chen, Z. N. Vapor- and mechanical-grinding-triggered color and luminescence switches for bis(sigma-fluorophenylacetylide) platinum(II) complexes. Chem. Eur. J. 17, 1171-1183 (2011)

24. Liou, G. S., Hsiao, S. H. \& Su, T. H. Synthesis, luminescence and electrochromism of aromatic poly(amine-amide)s with pendent triphenylamine moieties. J. Mater. Chem. 15, 1812-1820 (2005).

25. Sun, H. B. et al. Smart responsive phosphorescent materials for data recording and security protection. Nat. Commun. 5, 3601 (2014).

26. Diaz, R., Palleau, E., Poirot, D., Sangeetha, N. M. \& Ressier, L. High-throughput fabrication of anticounterfeiting colloid-based photoluminescent microtags using electrical nanoimprint lithography. Nanotechnology 25, 345302 (2014).

27. Ke, C. et al. Quantitative emergence of hetero[4] rotaxanes by template-directed click chemistry. Angew. Chem. Int. Ed. 52, 381-387 (2013).

28. Ke, C. et al. Pillar[5] arene as a co-factor in templating rotaxane formation. J. Am. Chem. Soc. 135, 17019-17030 (2013).

29. Hou, X. et al. Efficient syntheses of pillar[6] arene-based hetero[4]rotaxanes using a cooperative capture strategy. Chem. Commun. 50, 6196-6199 (2014).

30. Slamaschwok, A. et al. Interactions of the dimethyldiazaperopyrenium dication with nucleic-acids. 1. Binding to nucleic-acid components and to singlestranded polynucleotides and photocleavage of single-stranded oligonucleotides. Biochemistry 28, 3227-3234 (1989).

31. Slamaschwok, A. et al. Interactions of the dimethyldiazaperopyrenium dication with nucleic-acids. 2. Binding to double-stranded polynucleotides. Biochemistry 28, 3234-3242 (1989).

32. Biedermann, F., Elmalem, E., Ghosh, I., Nau, W. M. \& Scherman, O. A. Strongly fluorescent, switchable perylene bis(diimide) host-guest complexes with cucurbit[8] uril in water. Angew. Chem. Int. Ed. 51, 7739-7743 (2012).

33. Biedermann, F., Uzunova, V. D., Scherman, O. A., Nau, W. M. \& De Simone, A. Release of high-energy water as an essential driving force for the high-affinity binding of Cucurbit[n]urils. J. Am. Chem. Soc. 134, 15318-15323 (2012).

34. Seibt, J. et al. On the geometry dependence of molecular dimer spectra with an application to aggregates of perylene bisimide. Chem. Phys. 328, 354-362 (2006).

35. Shao, C. Z., Grune, M., Stolte, M. \& Würthner, F. Perylene bisimide dimer aggregates: fundamental insights into self-assembly by NMR and UV/Vis spectroscopy. Chem. Eur. J. 18, 13665-13677 (2012).

36. Whitty, A. Cooperativity and biological complexity. Nat. Chem. Biol. 4, 435-439 (2008)

37. Hunter, C. A. \& Anderson, H. L. What is cooperativity? Angew. Chem. Int. Ed. 48, 7488-7499 (2009).

38. Ercolani, G. \& Schiaffino, L. Allosteric, chelate, and interannular cooperativity: a mise au point. Angew. Chem. Int. Ed. 50, 1762-1768 (2011).

39. Zhang, X., Rehm, S., Safont-Sempere, M. M. \& Würthner, F. Vesicular perylene dye nanocapsules as supramolecular fluorescent $\mathrm{pH}$ sensor systems. Nat. Chem. 1, 623-629 (2009).
40. Fennel, F. et al. Biphasic self-assembly pathways and size-dependent photophysical properties of perylene bisimide dye aggregates. J. Am. Chem. Soc. 135, 18722-18725 (2013).

41. Medintz, I. L. et al. Self-assembled nanoscale biosensors based on quantum dot FRET donors. Nat. Mater. 2, 630-638 (2003).

42. Rizzo, M. A., Springer, G. H., Granada, B. \& Piston, D. W. An improved cyan fluorescent protein variant useful for FRET. Nat. Biotechnol. 22, 445-449 (2004).

43. Kajtar, M., Horvathtoro, C., Kuthi, E. \& Szejtli, J. A simple rule for predicting circular-dichroism induced in aromatic guests by cyclodextrin hosts in inclusion complexes. Acta. Chim. Acad. Sci. Hung. 110, 327-355 (1982).

44. Kodaka, M. A general rule for circular-dichroism induced by a chiral macrocycle. J. Am. Chem. Soc. 115, 3702-3705 (1993).

45. Allenmark, S. Induced circular dichroism by chiral molecular interaction. Chirality 15, 409-422 (2003).

\section{Acknowledgements}

The authors dedicate this manuscript to Professor Yoshihisa Inoue on the occasion of his retirement. We thank Professor Yoshihisa Inoue from Osaka University and Professor Frank Würthner from Universität Würzburg for their useful suggestions. We acknowledge financial support from Northwestern University (NU). X.H. gratefully acknowledges support from the Ryan Fellowship and the Northwestern University International Institute of Nanotechnology.

\section{Author contributions}

C.K. and X.H. conceived the project, X.H. and C.K. performed the experiments and analysed the data under the direction of J.F.S., C.J.B. and P.R.M. gave suggestions to optimize the system, R.B.P. suggested the potential application as ink, and all authors contributed in the manuscript preparation.

\section{Additional information}

Supplementary Information accompanies this paper at http://www.nature.com/ naturecommunications

Competing financial interests: The authors declare no competing financial interests.

Reprints and permission information is available online at http://npg.nature.com/ reprintsandpermissions/

How to cite this article: Hou, X. et al. Tunable solid-state fluorescent materials for supramolecular encryption. Nat. Commun. 6:6884 doi: 10.1038/ncomms7884 (2015).

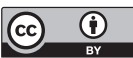

This work is licensed under a Creative Commons Attribution 4.0 International License. The images or other third party material in this article are included in the article's Creative Commons license, unless indicated otherwise in the credit line; if the material is not included under the Creative Commons license, users will need to obtain permission from the license holder to reproduce the material. To view a copy of this license, visit http://creativecommons.org/licenses/by/4.0/ 